\title{
DEJA VU ALL OVER AGAIN: THE REAPPEARANCE OF SUPERNOVA REFSDAL
}

\author{
P. L. Kelly ${ }^{1}$, S. A. Rodney ${ }^{2}$, T. Treu ${ }^{3,25}$, L.-G. Strolger ${ }^{4}$, R. J. Foley ${ }^{5,6}$, S. W. Jha ${ }^{7}$, J. Selsing ${ }^{8}$, G. Brammer ${ }^{4}$, M. BradaČ ${ }^{9}$, \\ S. B. Cenko ${ }^{10,11}$, O. Graur ${ }^{12,13}$, A. V. Filippenko ${ }^{1}$, J. Huorth ${ }^{8}$, C. McCully ${ }^{14,15}$, A. Molino ${ }^{16,17}$ M. Nonino $^{18}$, \\ ${ }^{1}$ Department of Astronomy, University of California, Berkeley, CA 94720-3411, USA; pkelly@astro.berkeley.edu \\ ${ }^{2}$ Department of Physics and Astronomy, University of South Carolina, 712 Main Street, Columbia, SC 29208, USA \\ ${ }^{3}$ Department of Physics and Astronomy, University of California, Los Angeles, CA 90095, USA \\ ${ }^{4}$ Space Telescope Science Institute, 3700 San Martin Drive, Baltimore, MD 21218, USA \\ ${ }_{6}^{5}$ Department of Physics, University of Illinois at Urbana-Champaign, 1110 W. Green Street, Urbana, IL 61801, USA \\ 6 Astronomy Department, University of Illinois at Urbana-Champaign, 1002 W. Green Street, Urbana, IL 61801, USA \\ ${ }^{7}$ Department of Physics and Astronomy, Rutgers, The State University of New Jersey, Piscataway, NJ 08854, USA \\ ${ }^{8}$ Dark Cosmology Centre, Niels Bohr Institute, University of Copenhagen, Juliane Maries Vej 30, DK-2100 Copenhagen, Denmark \\ ${ }^{9}$ University of California Davis, 1 Shields Avenue, Davis, CA 95616, USA \\ ${ }^{10}$ Astrophysics Science Division, NASA Goddard Space Flight Center, MC 661, Greenbelt, MD 20771, USA \\ 11 Joint Space-Science Institute, University of Maryland, College Park, MD 20742, USA \\ ${ }^{12}$ Center for Cosmology and Particle Physics, New York University, New York, NY 10003, USA \\ ${ }^{13}$ Department of Astrophysics, American Museum of Natural History, Central Park West and 79th Street, New York, NY 10024, USA \\ ${ }^{14}$ Las Cumbres Observatory Global Telescope Network, 6740 Cortona Drive, Suite 102, Goleta, California 93117, USA \\ ${ }^{15}$ Department of Physics, University of California, Santa Barbara, CA 93106-9530, USA \\ ${ }^{16}$ Instituto de Astronomia, Geofisica e Ciências Atmosféricas, Universidade de São Paulo, Cidade Universitária, 05508-090, São Paulo, Brazil \\ ${ }^{17}$ Instituto de Astrofísica de Andalucía (CSIC), E-18080 Granada, Spain \\ ${ }^{18}$ INAF, Osservatorio Astronomico di Trieste, via G.B. Tiepolo 11, I-40131 Trieste, Italy \\ ${ }^{19}$ Department of Physics and Astronomy, The Johns Hopkins University, 3400 N. Charles Street, Baltimore, MD 21218, USA \\ ${ }^{20}$ Leibniz-Institut für Astrophysik Potsdam (AIP), An der Sternwarte 16, D-14482 Potsdam, Germany \\ ${ }^{21}$ The Research School of Astronomy and Astrophysics, Australian National University, Mount Stromlo Observatory, \\ via Cotter Road, Weston Creek, Australian Capital Territory 2611, Australia
22 Physics and Astronomy Department, Stony Brook University, Stony Brook, NY 11794-3800, USA \\ ${ }^{23}$ Department of Astronomy, University of Arizona, Tucson, AZ 85721, USA \\ ${ }^{24}$ California Institute of Technology, 1200 East California Boulevard, Pasadena, CA 91125, USA \\ Received 2015 December 11; accepted 2016 February 6; published 2016 February 24
}

\begin{abstract}
In Hubble Space Telescope (HST) imaging taken on 2014 November 10, four images of supernova (SN) "Refsdal" (redshift $z=1.49$ ) appeared in an Einstein-cross-like configuration (images S1-S4) around an early-type galaxy in the cluster MACS J1149.5+2223 $(z=0.54)$. Almost all lens models of the cluster have predicted that the SN should reappear within a year in a second host-galaxy image created by the cluster's potential. In HST observations taken on 2015 December 11, we find a new source at the predicted position of the new image of SN Refsdal approximately $8^{\prime \prime}$ from the previous images S1-S4. This marks the first time the appearance of a SN at a particular time and location in the sky was successfully predicted in advance! We use these data and the light curve from the first four observed images of SN Refsdal to place constraints on the relative time delay and magnification of the new image (SX) compared to images S1-S4. This enables us, for the first time, to test "blind" lens model predictions of both magnifications and time delays for a lensed SN. We find that the timing and brightness of the new image are consistent with the blind predictions of a fraction of the models. The reappearance illustrates the discriminatory power of this blind test and its utility to uncover sources of systematic uncertainty. From planned $H S T$ photometry, we expect to reach a precision of $1 \%-2 \%$ on the time delay between S1-S4 and SX.
\end{abstract}

Key words: galaxies: clusters: general - galaxies: individual (MACS J1149.5+2223) - gravitational lensing: strong - supernovae: general - supernovae: individual (SN Refsdal)

\section{INTRODUCTION}

Background sources strongly lensed by galaxies and galaxy clusters that exhibit flux variations in time can be used as powerful probes, because they make it possible to measure the relative time delays between their multiple images. As Refsdal (1964) first suggested, time delays are useful because they depend sensitively on both the cosmic expansion rate and the gravitational potential of the lens. While the positions of the images of lensed galaxies depend on the derivative of the potential, time delays are directly proportional to differences in the potential.

\footnotetext{
${ }^{25}$ Packard Fellow.
}

Refsdal (1964) examined the utility of time-delay measurements of a strongly lensed, multiply imaged, resolved supernova (SN), but such an object was not found in the following five decades. A handful of $\mathrm{SNe}$ in galaxy-cluster fields have been magnified $(\sim 1.4-4 \times)$ by the cluster's potential (Goobar et al. 2009; Nordin et al. 2014; Patel et al. 2014; Rodney et al. 2015a), but none has been multiply imaged. A luminous H-poor SN at redshift $z=1.38$ (Chornock et al. 2013) was shown to be a highly magnified $(\sim 30 \times)$ SN Ia (Quimby et al. 2013, 2014), but the only existing exposures, taken from the ground, could not resolve multiple images of the SN.

Although strongly lensed SNe have eluded detection for 50 years, the discovery of multiply imaged quasars beginning in 1979 (Walsh et al. 1979) has made it possible to measure time 
delays for more than 20 systems (see, e.g., Kundic et al. 1997; Fassnacht et al. 1999; Tewes et al. 2013). For a subset of multiply imaged quasars with simple, early-type galaxy lenses, it has been possible to precisely predict the delay arising from the gravitational potential and thereby to measure an absolute distance scale and $H_{0}$ geometrically (e.g., Paraficz \& Hjorth 2010; Suyu et al. 2013, 2014). Although they are more difficult to find, strongly lensed SNe hold great promise as tools for time-delay cosmography (Kolatt \& Bartelmann 1998; Holz 2001; Bolton \& Burles 2003; Oguri \& Kawano 2003; Dobler \& Keeton 2006). In comparison with quasars light curves, those of $\mathrm{SNe}$ are relatively simple, and the peak luminosities of SNe Ia (Phillips 1993) and (with less precision) SNe IIP (e.g., Kirshner \& Kwan 1974) can be calibrated absolutely, thus providing a measurement of lensing magnification.

Kelly et al. (2015b) reported the discovery of SN Refsdal, the first strongly lensed SN resolved into multiple images, in the MACS J1149.5+2223 (Ebeling et al. 2001, 2007) galaxycluster field in Hubble Space Telescope (HST) images taken on 2014 November 10 (UT dates are used throughout this paper). Those exposures, collected as part of the Grism Lens-Amplified Survey from Space (GLASS; PI Treu; GO-13459; Schmidt et al. 2014; Treu et al. 2015), revealed four resolved images of the background $\mathrm{SNe}$ arranged in an Einstein cross configuration around an elliptical cluster member. Models of the complex potential of the galaxy cluster and early-type galaxy lens suggest that three of the four images are magnified by up to a factor of 10-20 (Grillo et al. 2015; Jauzac et al. 2016; Kawamata et al. 2015; Kelly et al. 2015b; Oguri 2015; Sharon \& Johnson 2015; Diego et al. 2016; Treu et al. 2016).

Models of the massive MACS J1149.5+2223 cluster $\left((1.4 \pm 0.3) \times 10^{15} M_{\odot}\right.$; Applegate et al. 2014; Kelly et al. 2014; von der Linden et al. 2014) produced soon after the discovery predicted that SN Refsdal would appear within several years in a different host-galaxy image close to the cluster core, $\sim 8^{\prime \prime}$ from images S1-S4 (Kelly et al. 2015b; Oguri 2015; Sharon \& Johnson 2015; Diego et al. 2016). We adopt the identifier "SX" for this new image, following Oguri (2015). Here we report the appearance of the image SX of SN Refsdal in HST images (PI: Kelly; GO-14199) taken on 2015 December 11.

In models of the MACS J1149.5+2223 cluster lens, the galaxy-cluster gravitational potential is constrained by varying combinations of strong-lensing constraints, including the positions and redshifts of multiply imaged background galaxies, the positions of the SN Refsdal images S1-S4, and locations of bright clumps within SN Refsdal's host galaxy. The potential (or surface mass density) is also parameterized in a variety of ways, often using the positions or light distributions of cluster galaxies as constraints. Given the complexity of the cluster potential, it is unlikely that measurement of time delays between the SN Refsdal images can be used for precision cosmology as suggested by Refsdal (1964). However, if one adopts a fixed set of cosmological parameters, then time delays and magnification ratios can be used to measure the difference in the potential and its derivatives between the positions of multiple images, thus providing a powerful local test of lens models.

To sharpen this test, several lens-modeling teams have refined their predictions for the relative time delay and magnification of image SX. Treu et al. (2016) identified an improved set of multiply imaged galaxies using additional data collected soon before and after the discovery of the SN. Systems were discovered or confirmed from HST WFC3 G102 and G141 grism spectra (PI Treu; GO-13459), 30 orbits of G141 grism spectra taken to determine the spectroscopic type of the SN (PI Kelly; GO-14041; Kelly et al. 2015a; G. Brammer et al. 2016, in preparation), deep VLT-MUSE observations (PI Grillo), Keck/DEIMOS observations (PI Jha), as well as Frontier Fields observations of the MACS J1149.5 +2223 field that began shortly after discovery (PI Lotz; GO13504). These data provided 429 spectroscopic redshifts in the field of MACS J1149.5+2223, including 170 cluster members and 23 multiple images of 10 different galaxies. With the improved data set, Treu et al. (2016) organized five independent lens-modeling teams that produced seven separate predictions for the time delay. In a parallel effort, Jauzac et al. (2016) used new Gemini GMOS and part of the VLT-MUSE data (PI Grillo), as well as Frontier Fields photometry, to generate improved constraints on the cluser potential and new predictions for the time delay and magnification of image SX.

These revised models largely favored delays of less than one year and predicted that image SX would be significantly fainter than images $\mathrm{S} 1-\mathrm{S} 3$ by a factor of 3-4. Together, these predictions indicated that image SX could plausibly have been detected as soon as HST could observe the MACS J1149.5 +2223 field beginning on 2015 October 30. From late July through late October, it had been too close to the Sun to be observed. Importantly, all of these modeling efforts were completed before the first realistic opportunity to detect image SX on 2015 October 30, making these truly blind predictions.

Here we present a direct test of the lens model predictions, as we revisit the MACS J1149.5+2223 field and identify the appearance of the anticipated fifth image of SN Refsdal. In Kelly et al. (2015a), we classify SN Refsdal as the explosion of an H-rich compact massive star broadly similar to SN 1987A, and in Rodney et al. (2015b) we measure time delays between images S1 through S4. Section 2 in this paper presents the data processing and photometry of the new HST images. In Section 3, we derive joint constraints on the relative time delay and magnification of image SX and compare these to the published predictions from the lens-modeling community. We briefly discuss our results in Section 4 and conclude in Section 5. Throughout this paper, magnitudes are given in the AB system (Oke \& Gunn 1983), and a concordance cosmology is assumed when necessary $\left(\Omega_{m}=0.3, \Omega_{\Lambda}=0.7\right.$, $H_{0}=70 \mathrm{~km} \mathrm{~s}^{-1} \mathrm{Mpc}^{-1}$ ).

\section{METHODS}

We processed the WFC3 imaging data using a pipeline constructed from the DrizzlePac software tools. ${ }^{26}$ The images were resampled to a scale of $0 !^{\prime \prime} 06 \mathrm{pix}^{-1}$ using AstroDrizzle (Fruchter et al. 2010) and then registered to a common astrometric frame using TweakReg. Template images in each band were constructed by combining all available WFC3 infrared (IR) imaging collected prior to 2015 October 30, comprising observations from the GLASS program, the Cluster Lensing And Supernova survey with Hubble (CLASH; GO-12068; PI M. Postman; Postman et al. 2012), the Hubble Frontier Fields (HFF; DD/GO-13504; PI J. Lotz), the FrontierSN program (GO-13790; PI S. Rodney), and

${ }^{26}$ http://drizzlepac.stsci.edu 
Table 1

Measurements of Image SX

\begin{tabular}{lclcc}
\hline \hline Obs. Date & & Filter & $\begin{array}{c}\text { Exp. Time } \\
(\mathrm{s})\end{array}$ & $\begin{array}{c}\text { Magnitude } \\
(\mathrm{AB})\end{array}$ \\
\hline 2015 Oct 30 & 57325.8 & F125W & 1259 & $27.4 \pm 0.4$ \\
2015 Nov 14 & 57340.9 & F125W & 1259 & $27.3 \pm 0.4$ \\
2015 Dec 11 & 57367.1 & F125W & 1259 & $26.56 \pm 0.16$ \\
2015 Oct 30 & 57325.9 & F160W & 1159 & $27.4 \pm 0.6$ \\
2015 Nov 14 & 57341.0 & F160W & 1159 & $26.29 \pm 0.15$ \\
2015 Dec 11 & 57367.1 & F160W & 1159 & $26.24 \pm 0.16$ \\
\hline
\end{tabular}

Note. The fluxes are measured at the position of image SX. Photometry of the images obtained on 2015 December 11 yields a $F 125 \mathrm{~W}-\mathrm{F} 160 \mathrm{~W}$ color of $0.2 \pm 0.3 \mathrm{mag} \mathrm{AB}$. The coordinates of image $\mathrm{SX}$ are $\alpha=11^{\mathrm{h}} 49^{\mathrm{m}} 36^{\mathrm{s}} .02$, $\delta=+22^{\circ} 23^{\prime} 48^{\prime \prime} .1(\mathrm{~J} 2000.0)$.

the SN Refsdal Follow-up program (DD/GO-14041; PI P. Kelly). The excellent stability of the HST point-spread function (PSF) allowed us to generate difference images by simply subtracting these archival template images directly from the search-epoch images.

To measure the SN flux from the difference images, we used the PythonPhot ${ }^{27}$ software package (Jones et al. 2015) which implements PSF fitting based on the DAOPHOT algorithm (Stetson 1987). We measure photometric uncertainties by planting and recovering 1000 fake stars (copies of a model PSF) in the vicinity of the SN position.

A principal inference we make is to constrain the time delay and magnification of image SX relative to image $\mathrm{S} 1$. We simulate potential light curves of image SX by shifting the light curve of image $\mathrm{S} 1$ in time and demagnifying it. To construct a simple model of the light curve of image S1, we fit separate second-order polynomials to its $F 125 \mathrm{~W}$ and $F 160 \mathrm{~W}$ flux measurements (see Rodney et al. 2015b and Kelly et al. 2015a). We next calculate the expected brightness in $F 125 \mathrm{~W}$ and F160W of image SX at each epoch listed in Table 1 across a grid of time delays and magnifications. The combined probability of the measured fluxes is taken to be the likelihood of each pair of relative time delay and magnification values. While we use the light curve of image S1 as a model, those of $\mathrm{S} 2$ and $\mathrm{S} 3$ yield almost identical constraints.

\section{RESULTS}

In Figure 1, we present a coaddition of the $F 125 \mathrm{~W}$ and F160W images taken on 2015 December 11 which shows the reappearance of SN Refsdal in a different image of its $z=1.49$ host galaxy. The coordinates of this image SX are $\alpha=11^{\mathrm{h}} 49^{\mathrm{m}} 36 . \mathrm{s} 02, \delta=+22^{\circ} 23^{\prime} 48^{\prime \prime} 1$ (J2000.0). ${ }^{28}$ This locates SX at 6." 2 north and 3 ". 9 east of image S1. Table 1 reports the measured fluxes and uncertainties. We find a $F 125 \mathrm{~W}-\mathrm{F} 160 \mathrm{~W}$ color of $0.2 \pm 0.3 \mathrm{mag} \mathrm{AB}$ for image $\mathrm{SX}$, consistent with those measured for the four $\mathrm{SN}$ images forming the Einstein cross at discovery. We detect a fainter source at the same coordinates in a combination of the $F 125 \mathrm{~W}$ and $F 160 \mathrm{~W}$ images taken on 2015 November 14, while the 2015 October 30 images yield no statistically significant detection (see Table 1).

\footnotetext{
27 https://github.com/djones1040/PythonPhot

28 The coordinates are registered to the astrometric system used for the CLASH, GLASS, and HFF images and catalogs, http://www.stsci.edu/hst/ campaigns/frontier-fields/.
}

Images S1-S3 of SN Refsdal remain visible in the coaddition of images taken on 2015 December 11.

Figure 2 shows a comparison between the coordinates of the new image SX and several published model predictions, which are in good agreement. In Figure 3, we plot simultaneous constraints on the time delay and magnification ratio between image S1 discovered in 2014 and the newly detected image SX. We show model predictions for the relative time delay and magnification between SX and S1 from several teams reported by Treu et al. (2016) as well as independent predictions by Jauzac et al. (2016).

While the other plotted predictions were made blind to the observations beginning on 2015 October 30, the two predictions plotted in Figure 3 with dashed lines, "Zitrin-c" and "Jauzac-s," were updated at a later date; hence, these last two are not truly blind predictions, although they incorporate no additional data. "Zitrin-c," an improvement over the "Zitrin-g" model, allows the early-type lens galaxy to be freely weighted to assure that the critical curves pass between the four Einstein cross images. In the case of "Jauzac-s," the authors addressed an issue with the use of LENSTOOL (Kneib et al. 1996; Jullo et al. 2007) for computing time delays. LENSTOOL allows multiple images of a strongly lensed object such as SN Refsdal to map to differing positions on the source plane, which can translate into an aberration of the predicted relative time delays. For "Jauzac-s," the authors estimate a single, common position for SN Refsdal in the source plane and analytically estimate improved model time delays using this common position (see Section 5.2.2 of Jauzac et al. 2016).

\section{DISCUSSION}

Lensed SNe provide a powerful means to test the accuracy of the lens models of the foreground deflector, or to provide additional input constraints (e.g., Riehm et al. 2011). Previous tests have been based on $\mathrm{SNe}$ that are magnified but not multiply imaged (Nordin et al. 2014; Patel et al. 2014). Recently, Rodney et al. (2015a) discovered a SN Ia magnified by a factor of $\sim 2$ by a galaxy-cluster potential and found that its calibrated luminosity was in tension with some-but not allmodels of the cluster potential.

With SN Refsdal we have for the first time been able to test predictions for both the lensing time delay and the magnification. This is important because the time delay depends on the difference in gravitational potential, while magnification depends on a combination of second derivatives, and therefore the two observables test different aspects of the potential. In principle, time delays are much less sensitive than magnification ratios to millilensing and microlensing; they should therefore be more robustly predicted.

It is important to keep in mind that all of these tests are local, and thus a larger sample is needed to assess the global goodness of fit of every model. Nevertheless, these tests are an extremely valuable probe of systematics. In fact, as discussed by Treu et al. (2016), the uncertainties reported by modelers do not include all sources of systematic errors. For example, systematic uncertainties arising from unmodeled millilensing, residual mass-sheet degeneracy, and multiplane lensing are very difficult to calculate and are thus not included. The lensedSN tests provide estimates of the amplitude of the unknown uncertainties. Other known sources of errors are not included either. For example, a 3\% uncertainty in the Hubble constant (Riess et al. 2011) implies a 3\% uncertainty in time delays (i.e., 

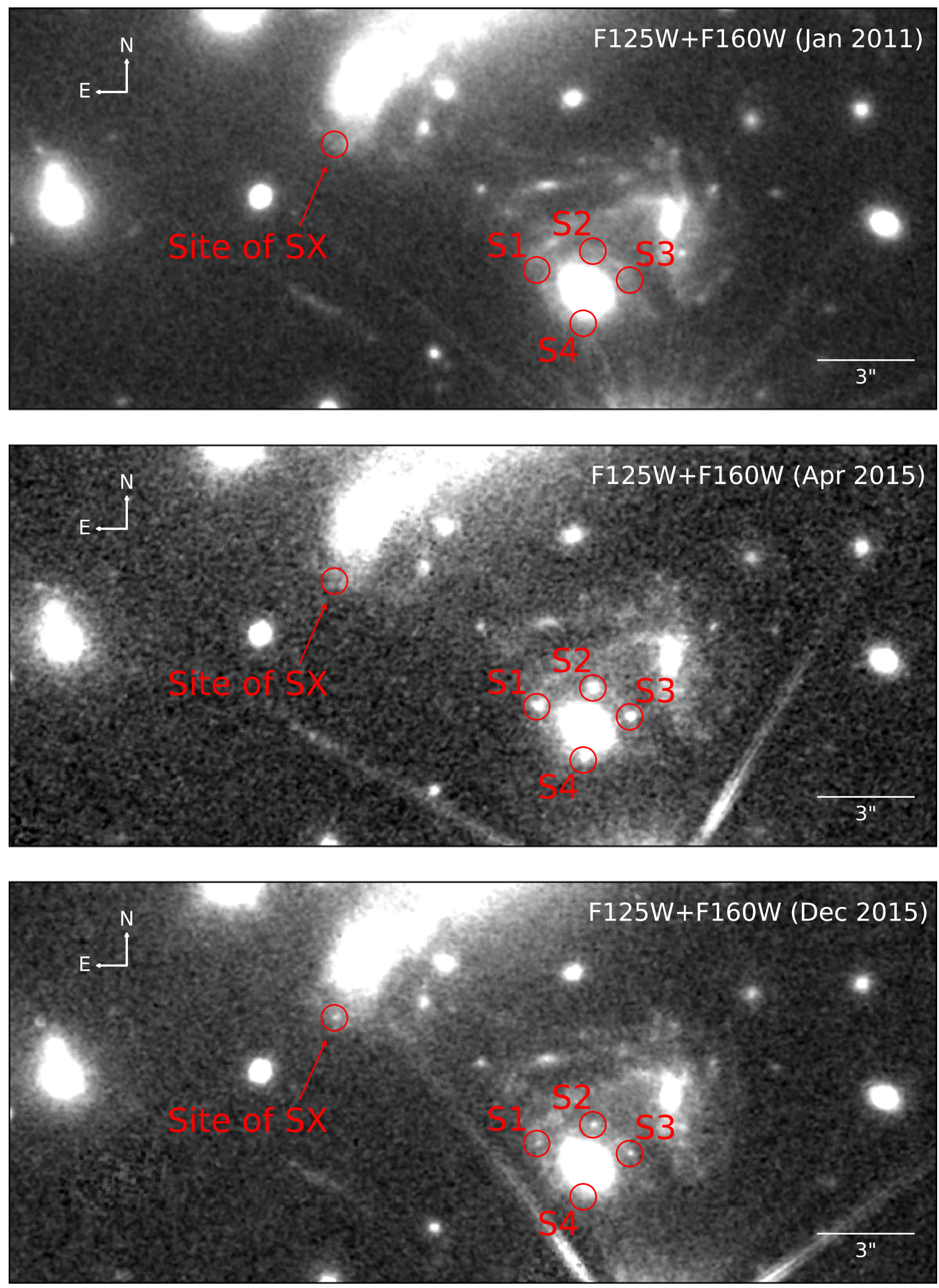

Figure 1. Coadded WFC3-IR F125W and F160W exposures of the MACS J1149.5+2223 galaxy-cluster field taken with HST. The top panel shows images acquired in 2011 before the SN appeared in S1-S4 or SX. The middle panel displays images taken on 2015 April 20 when the four images forming the Einstein cross are close to maximum brightness, but no flux is evident at the position of SX. The bottom panel shows images taken on 2015 December 11 which reveal the new image SX of SN Refsdal. Images S1-S3 in the Einstein cross configuration remain visible in the 2015 December 11 coadded image (see Kelly et al. 2015a and Rodney et al. 2015b for analysis of the SN light curve). 


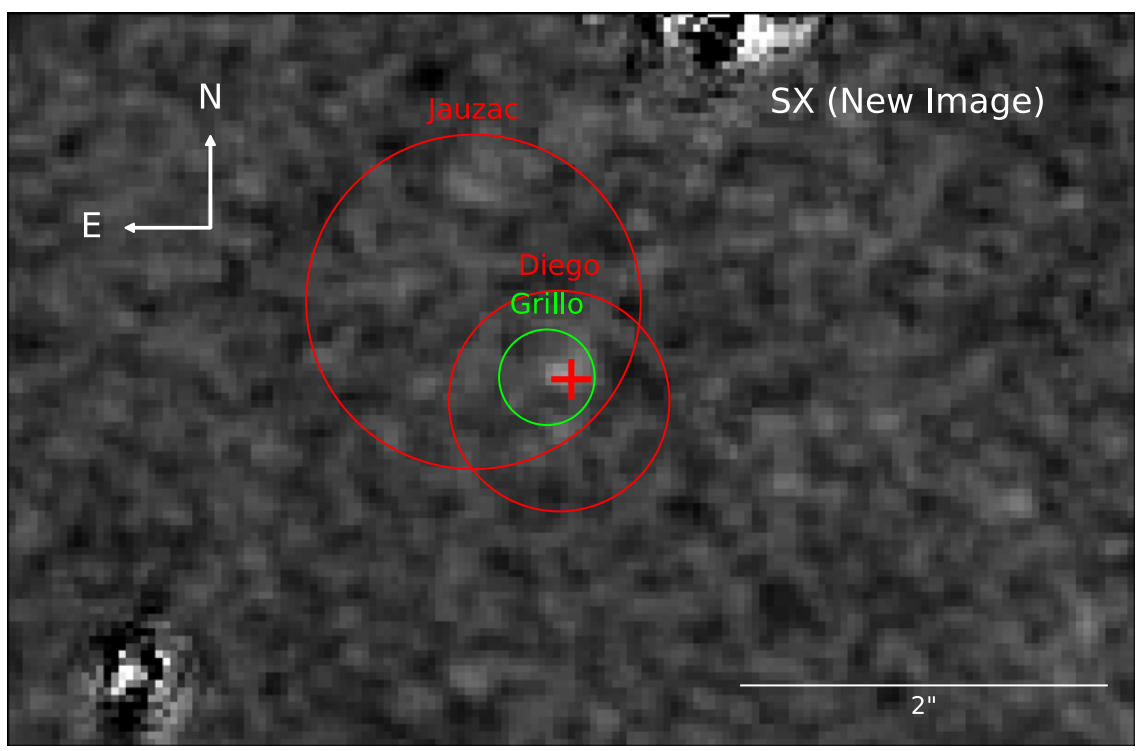

Figure 2. Comparison between the predicted and the actual position of image SX of SN Refsdal. Coordinate published predictions are overplotted on the coaddition of $F 125 \mathrm{~W}$ and $F 160 \mathrm{~W}$ difference images made by subtracting the 2015 December 11 exposures from archival template images taken in 2011 . The circles show the rms of the angular offsets between the measured positions of multiply imaged sources and their positions in the best-fitting respective models. The Diego et al. (2016), Jauzac et al. (2016), and Grillo et al. (2015) predictions are all consistent with the measured position of image SX within the reported rms scatter. The residual scatter for the Diego et al. (2016) model was not published and is 0" 6 (J. Diego 2015, private communication).

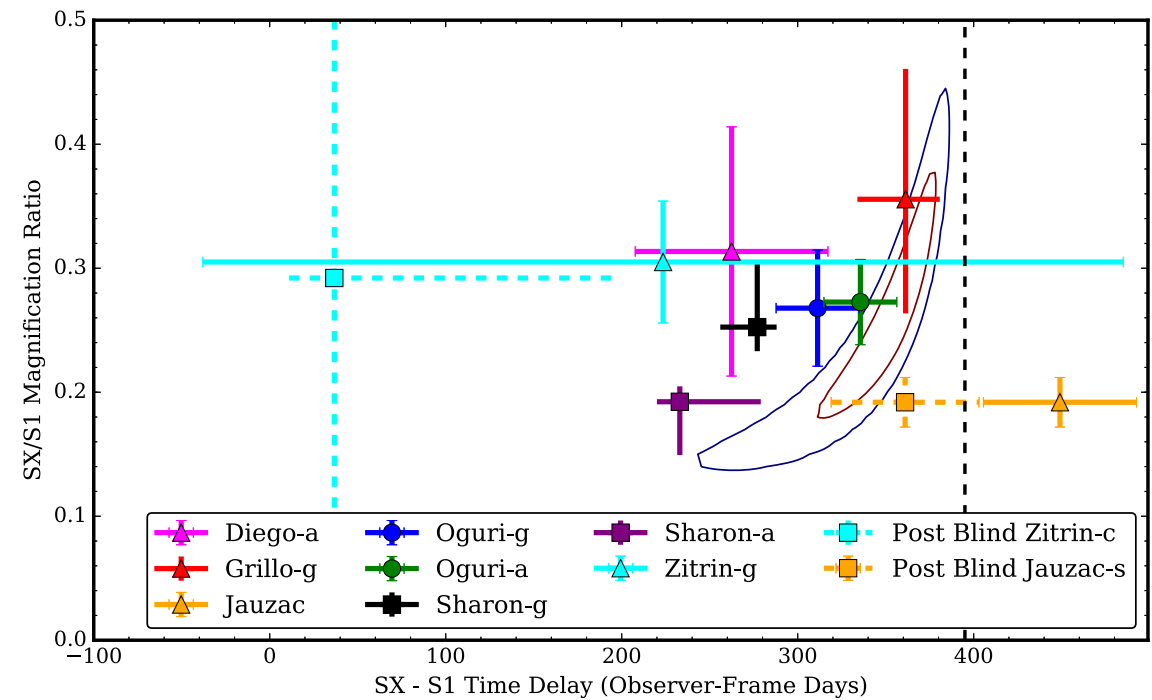

Figure 3. Simultaneous constraints on the time delay and magnification of image SX relative to image S1 from photometry of image SX listed in Table 1. The twodimensional contours show the $68 \%$ and $95 \%$ confidence levels, and model predictions plot $68 \%$ confidence levels. Since many of the lensing predictions are not Gaussian distributed, the $68 \%$ limits do not imply that they are necessarily inconsistent with the measurements. Except for the Jauzac et al. (2016) prediction, labels refer to models presented by Treu et al. (2016). While all other plotted predictions were made in advance of the HST Cycle 23 observations beginning on 2015 October 30, "Post Blind Zitrin-c" and "Post Blind Jauzac" were updates made at a later date. "Post Blind Zitrin-c" is an update of the "Zitrin-g" model where the lens galaxy was left to be freely weighted to assure that its critical curves pass between the four Einstein cross images. For "Post Blind Jauzac," the authors compute a common position for images S1-S4 in the source plane and recompute the time delays analytically using their LENSTOOL model of the cluster potential. The greater the S1-SX delay, the earlier the 2015 December 11 observations are in the light curve of SX. The black dashed line marks the delay beyond which we lack data on the light curve of SN Refsdal. We extrapolate to earlier epochs using the best-fitting second-order polynomials.

$\sim 10$ days for a year-long delay). Furthermore, the uncertainties are typically highly non-Gaussian, so the $95 \%$ confidence interval is not simply twice as wide as the $68 \%$ one.

\section{CONCLUSIONS}

With models of the MACS J1149.5+2223 galaxy-cluster potential, the appearance of SN Refsdal in November of 2014 as an Einstein cross became an augury of its future arrival $\sim 8^{\prime \prime}$ away in a different image of its host galaxy. The detection of the reappearance here shows the power of modern-day predictions using models of the distribution of matter in galaxy clusters and the general theory of relativity. The timing and brightness of light from SNRefsdal in image SX is approximately in agreement with predictions, implying that, for most models, unknown systematic uncertainties cannot be substantially larger than random uncertainties. At the same time, this first detection provides some discriminatory power: not all models fare equally well. Grillo-g, Oguri-g, Oguri-a, and Sharon-a appear to be the ones that match the observations 
most closely. In general, most models seem to predict a slightly higher magnification ratio than observed, or shorter delays.

From the light curves of images S1-S4 of SN Refsdal, we can already anticipate how the brightness of image SX will evolve. An HST imaging program will continue to measure the light curve of image SX past peak brightness through 2017 (PI Kelly; GO-14199) ${ }^{29}$ and constrain the time delay and magnification of image SX relative to images S1-S4 to within $1 \%-2 \%$. These measurements will make it possible to discriminate among the model predictions with improved precision.

We express our appreciation for the efforts of Program Coordinator Beth Periello and Contact Scientist Norbert Pirzkal of STScI. Support for the analysis in this paper is from $H S T$ grant GO-14041. The GLASS program is supported by GO13459, and the FrontierSN photometric follow-up program has funding through GO-13386. A.Z. is supported by Hubble Fellowship (HF2-51334.001-A) awarded by STScI, which is operated for NASA by the Association of Universities for Research in Astronomy, Inc. under contract NAS 5-26555. R.J.F. gratefully acknowledges support from NSF grant AST1518052 and the Alfred P. Sloan Foundation. A.V.F.'s group at UC Berkeley has received generous financial assistance from the Christopher R. Redlich Fund, the TABASGO Foundation, and NSF grant AST-1211916. M.N. acknowledges PRININAF 2014 1.05.01.94.02. This supernova research at Rutgers University is supported by NSF CAREER award AST0847157, as well as NASA/Keck JPL RSA 1508337 and 1520634, to S.W.J.

\section{REFERENCES}

Applegate, D. E., von der Linden, A., Kelly, P. L., et al. 2014, MNRAS, 439, 48

Bolton, A. S., \& Burles, S. 2003, ApJ, 592, 17

Chornock, R., Berger, E., Rest, A., et al. 2013, ApJ, 767, 162

Diego, J. M., Broadhurst, T., Chen, C., et al. 2016, MNRAS, 456, 356

Dobler, G., \& Keeton, C. R. 2006, ApJ, 653, 1391

Ebeling, H., Barrett, E., Donovan, D., et al. 2007, ApJL, 661, L33

Ebeling, H., Edge, A. C., \& Henry, J. P. 2001, ApJ, 553, 668

Fassnacht, C. D., Pearson, T. J., Readhead, A. C. S., et al. 1999, ApJ, 527, 498
Fruchter, A. S., Hack, W., Dencheva, M., Droettboom, M., \& Greenfield, P. 2010, in 2010 Space Telescope Science Institute Calibration Workshop (Baltimore, MD: STScI), 382

Goobar, A., Paech, K., Stanishev, V., et al. 2009, A\&A, 507, 71

Grillo, C., Karman, W., Suyu, S. H., et al. 2015, ApJ, submitted (arXiv:1511.04093)

Holz, D. E. 2001, ApJL, 556, L71

Jauzac, M., Richard, J., Limousin, M., et al. 2016, MNRAS, 457, 2029

Jones, D. O., Scolnic, D. M., \& Rodney, S. A. 2015, Astrophysics Source Code Library, record ascl:1104.011

Jullo, E., Kneib, J.-P., Limousin, M., et al. 2007, NJPh, 9, 447

Kawamata, R., Oguri, M., Ishigaki, M., Shimasaku, K., \& Ouchi, M. 2015, ApJ, in press (arXiv:1510.06400)

Kelly, P. L., Brammer, G., Selsing, J., et al. 2015a, ApJ, submitted (arXiv:1512.09093)

Kelly, P. L., Rodney, S. A., Treu, T., et al. 2015b, Sci, 347, 1123

Kelly, P. L., von der Linden, A., Applegate, D. E., et al. 2014, MNRAS, 439, 28

Kirshner, R. P., \& Kwan, J. 1974, ApJ, 193, 27

Kneib, J.-P., Ellis, R. S., Smail, I., Couch, W. J., \& Sharples, R. M. 1996, ApJ, 471,643

Kolatt, T. S., \& Bartelmann, M. 1998, MNRAS, 296, 763

Kundic, T., Turner, E. L., Colley, W. N., et al. 1997, ApJ, 482, 75

Nordin, J., Rubin, D., Richard, J., et al. 2014, MNRAS, 440, 2742

Oguri, M. 2015, MNRAS, 449, L86

Oguri, M., \& Kawano, Y. 2003, MNRAS, 338, L25

Oke, J. B., \& Gunn, J. E. 1983, ApJ, 266, 713

Paraficz, D., \& Hjorth, J. 2010, ApJ, 712, 1378

Patel, B., McCully, C., Jha, S. W., et al. 2014, ApJ, 786, 9

Phillips, M. M. 1993, ApJL, 413, L105

Postman, M., Coe, D., Benítez, N., et al. 2012, ApJS, 199, 25

Quimby, R. M., Oguri, M., More, A., et al. 2014, Sci, 344, 396

Quimby, R. M., Werner, M. C., Oguri, M., et al. 2013, ApJL, 768, L20

Refsdal, S. 1964, MNRAS, 128, 307

Riehm, T., Mörtsell, E., Goobar, A., et al. 2011, A\&A, 536, A94

Riess, A. G., Macri, L., Casertano, S., et al. 2011, ApJ, 730, 119

Rodney, S. A., Patel, B., Scolnic, D., et al. 2015a, ApJ, 811, 70

Rodney, S. A., Strolger, L.-G., Kelly, P. L., et al. 2015b, ApJ, in press (arXiv:1512.05734)

Schmidt, K. B., Treu, T., Brammer, G. B., et al. 2014, ApJL, 782, L36

Sharon, K., \& Johnson, T. L. 2015, ApJL, 800, L26

Stetson, P. B. 1987, PASP, 99, 191

Suyu, S. H., Auger, M. W., Hilbert, S., et al. 2013, ApJ, 766, 70

Suyu, S. H., Treu, T., Hilbert, S., et al. 2014, ApJL, 788, L35

Tewes, M., Courbin, F., Meylan, G., et al. 2013, A\&A, 556, A22

Treu, T., Brammer, G., Diego, J. M., et al. 2016, ApJ, 817, 60

Treu, T., Schmidt, K. B., Brammer, G. B., et al. 2015, ApJ, 812, 114

von der Linden, A., Allen, M. T., Applegate, D. E., et al. 2014, MNRAS, 439, 2

Walsh, D., Carswell, R. F., \& Weymann, R. J. 1979, Natur, 279, 381

\footnotetext{
29 Indeed, as we will report in detail elsewhere, $H S T$ data obtained in January 2016 show SX brightening.
} 